DOI: https://doi.org/10.34069/AI/2022.49.01.25

How to Cite:

D’Elia Oliveira, L.E., Dutok-Sánchez, C.M., Balieiro Monteiro, R., de Medeiros, F.A. (2022). Aplicação de Annona glabra L. (Annonaceae) na área da saúde, composição química e atividade biológica. Amazonia Investiga, 11(49), 224-238. https://doi.org/10.34069/AI/2022.49.01.25

\title{
Aplicação de Annona glabra L. (Annonaceae) na área da saúde, composição química e atividade biológica
}

\section{Application of Annona glabra L. (Annonaceae) in the area of health, chemical composition and biological activity}

\section{Aplicación de Annona glabra L. (Annonaceae) en el área de salud, composición química y actividad biológica}

Luiz Eduardo D'Elia Oliveira ${ }^{99}$ https://orcid.org/0000-0003-4215-1598

Carlos Manuel Dutok-Sánchez ${ }^{100}$

https://orcid.org/0000-0001-6712-3185

Romário Balieiro Monteiro ${ }^{101}$

https://orcid.org/0000-0002-5986-3447

Fernando Antônio de Medeiros ${ }^{102}$

https://orcid.org/0000-0003-0588-1858

\section{Resumo}

Este artigo de revisão teve como objetivo apresentar o conhecimento acumulado até o presente momento a respeito da espécie Annona glabra L. (Annonaceae), sua aplicabilidade na área da saúde, composição química e atividade biológica. A seleção dos artigos ocorreu por meio de diferentes plataformas de busca bibliográfica, como Annual Reviews, Europe PMC, Google Acadêmico, Indian Journals, PubMed, ResearchGate, SAGE journals, Scielo, ScienceDirect, Scientific Reports, Semantic Scholar, Wiley Online Library e J-STAGE. A literatura científica apresenta estudos sobre a espécie $A$. glabra apresentam dados promissores sobre atividades anticarcinogênica, inibição das células carcinogênicas, leucemia linfocítica crônica e do complexo mitocondrial I, antioxidantes, antirreumática, emoliente, significativa inibição da replicação do HIV em linfócitos H9 e na inibição da transcriptase reversa, antimicrobiana, antifúngica, inseticida, vermicida e antiparasitária. Estudos fitoquímicos derivados das extrações de diferentes partes da planta revelaram a presença de flavonóides, terpenóides, glicosídeos, esteróides, saponinas,

\begin{abstract}
This review article aimed to present the knowledge accumulated so far about the species Annona glabra L. (Annonaceae), its applicability in the health area, chemical composition and biological activity. The selection of articles took place through different bibliographic search platforms, such as Annual Reviews, Europe PMC, Google Scholar, Indian Journals, PubMed, ResearchGate, SAGE journals, Scielo, ScienceDirect, Scientific Reports, Semantic Scholar, Wiley Online Library and J- STAGE The scientific literature presents studies on the species A. glabra present promising data on anticarcinogenic activities, inhibition of carcinogenic cells, chronic lymphocytic leukemia and mitochondrial complex I, antioxidants, antirheumatic, emollient, significant inhibition of HIV replication in $\mathrm{H} 9$ lymphocytes and inhibition of reverse transcriptase, antimicrobial, antifungal, insecticidal, vermicide and antiparasitic. Phytochemical studies derived from extractions of different parts of the plant revealed the presence of flavonoids, terpenoids, glycosides, steroids, saponins, tannins, anthraquinones and acidic compounds. The species has properties related to traditional medicine, being used in the Chinese, Japanese and Mexican health systems. The
\end{abstract}

\footnotetext{
${ }^{99}$ Programa de Pós-Graduação em Ciências da Saúde, Universidade Federal do Amapá, Rodovia Juscelino Kubitschek, Brasil.

${ }^{100}$ Curso de Enfermagem Campus Binacional do Oiapoque, Universidade Federal do Amapá, Bairro Universidade, Oiapoque, Brasil. Centro Universitário Leonardo da Vinci, Brasil.

${ }^{101}$ Curso de Licenciatura em Química, Universidade Federal do Amapá, Rodovia Juscelino Kubitschek, Brasil.

102 Programa de Pós-Graduação em Ciências da Saúde, Universidade Federal do Amapá, Rodovia Juscelino Kubitschek, Brasil.
} 


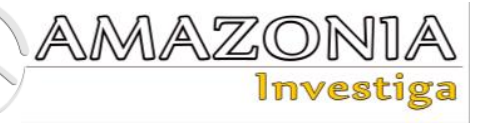

taninos, antraquinonas e compostos ácidos. A espécie apresenta propriedades relacionadas a medicina tradicional, sendo empregues no sistema de saúde chinês, japonês e mexicano. A compreensão acerca da espécie e seus compostos com atividade anticarcinogênica ainda é mal compreendida e infelizmente nenhum estudo alcançou a fase de testagem clínica. Este estudo buscou ser fonte de estímulo para novas pesquisas abordando a atividade biológica, estudos in vitro e in vivo, das diferentes peças florais de A. glabra.

Palavras-chave: Annonaceae, Annona glabra, Saúde, Compostos Químicos, Atividade Biológica.

\section{Resumen}

Este artículo de revisión tuvo como objetivo presentar el conocimiento acumulado hasta la fecha sobre la especie Annona glabra L. (Annonaceae), su aplicabilidad en el área de la salud, composición química y actividad biológica. La selección de artículos se realizó a través de diferentes plataformas de búsqueda bibliográfica, como Annual Reviews, Europe PMC, Academic Google, Indian Journals, PubMed, ResearchGate, SAGE journals, Scielo, ScienceDirect, Scientific Reports, Semantic Scholar, Wiley Online Library y J-STAGE. La literatura científica muestra estudios sobre la especie A. glabra que presentan datos prometedores sobre actividades anticancerígenas, inhibición de células cancerígenas, leucemia linfocítica crónica y complejo mitocondrial I, antioxidante, antirreumático, emoliente, inhibición significativa de la replicación del VIH en linfocitos H9 y en la inhibición de la transcriptasa inversa, antimicrobiano, antifúngico, insecticida, vermicida y antiparasitario. Los estudios fitoquímicos derivados de extracciones de diferentes partes de la planta revelaron la presencia de flavonoides, terpenoides, glucósidos, esteroides, saponinas, taninos, antraquinonas y compuestos ácidos. La especie tiene propiedades relacionadas con la medicina tradicional, siendo utilizada en el sistema de salud chino, japonés y mexicano. El conocimiento sobre la especie y sus compuestos con actividad anticancerígena aún es poco conocido y, lamentablemente, ningún estudio ha llegado a la etapa de prueba clínica. Este estudio buscó ser una fuente de estímulo para futuras investigaciones sobre la actividad biológica, estudios in vitro e in vivo, de diferentes piezas florales de A. glabra.

Palabras clave: Annonaceae, Annona glabra, Salud, Compuestos químicos, Actividad biológica.

\section{Introdução}

A espécie Annona glabra L. (Annonaceae) (Figura 1), é uma planta com ampla distribuição, principalmente nas regiões tropicais e subtropicais (Zotz, Tyree \& Patiño, 1997). Encontrada nas margens de lagos e rios, se desenvolvem em ambientes sujeitos a inundações podendo durar por períodos curtos, longos ou até permanecendo constantemente inundados (Campbell, Stone \& Rosas, 1992; Croat, 1978). Também ocorrendo em habitats pantanosos de manguezais, são tolerantes a água salgada ou salobra, não sendo capazes de sobreviver em terrenos com escassez hídrica, solos arenosos e bem drenados (Goodrich \& Raguso, 2009; Matsumoto et al., 2014; Zotz, Tyree, \& Patiño, 1997) e quando expostas a condições de seca suas raízes rapidamente são afetadas (Goodrich \& Raguso, 2009; Zotz et al., 1997).
A espécie A. glabra apresenta aplicações na área da saúde com propriedades relacionadas à fitoquímica e na medicina tradicional, os extratos crus são empregues no sistema de saúde chinês, japonês e mexicano (Cochrane et al., 2008); em relação a atividade biológica, nos frutos encontram-se compostos (acetogeninas, entcauranos, peptídeos e alcalóides) com inúmeras propriedades clínicas e farmacológicas em seres humanos (Anh et al., 2014).

Algumas das propriedades ligadas à espécie são relacionadas a sua ação inseticida (Chang et al., 2000; de Mendonça et al., 2005; Ohsawa et al., 1991; Padmaja et al., 1995; Zeng et al., 1996); antimicrobiana (Galvão et al., 2016; Padmaja et al., 1995; Zeng et al., 1996); antifúngica (Padmaja et al., 1995); antiparasitária (Balbach, 
1995; Ohsawa et al., 1991); antirreumática e emoliente (Balbach, 1995); inibição das células carcinogênicas, leucemia linfocítica crônica e do complexo mitocondrial I (Cochrane et al., 2008; Li et al., 1998; Liu et al., 1998; Matsumoto et al.,
2014); antioxidantes (Gonçalves et al., 2010; Genovese et al., 2008); significativa inibição da replicação do HIV em linfócitos H9 e na inibição do transcriptase reversa (HIV-1) (Chang et al., 1998).
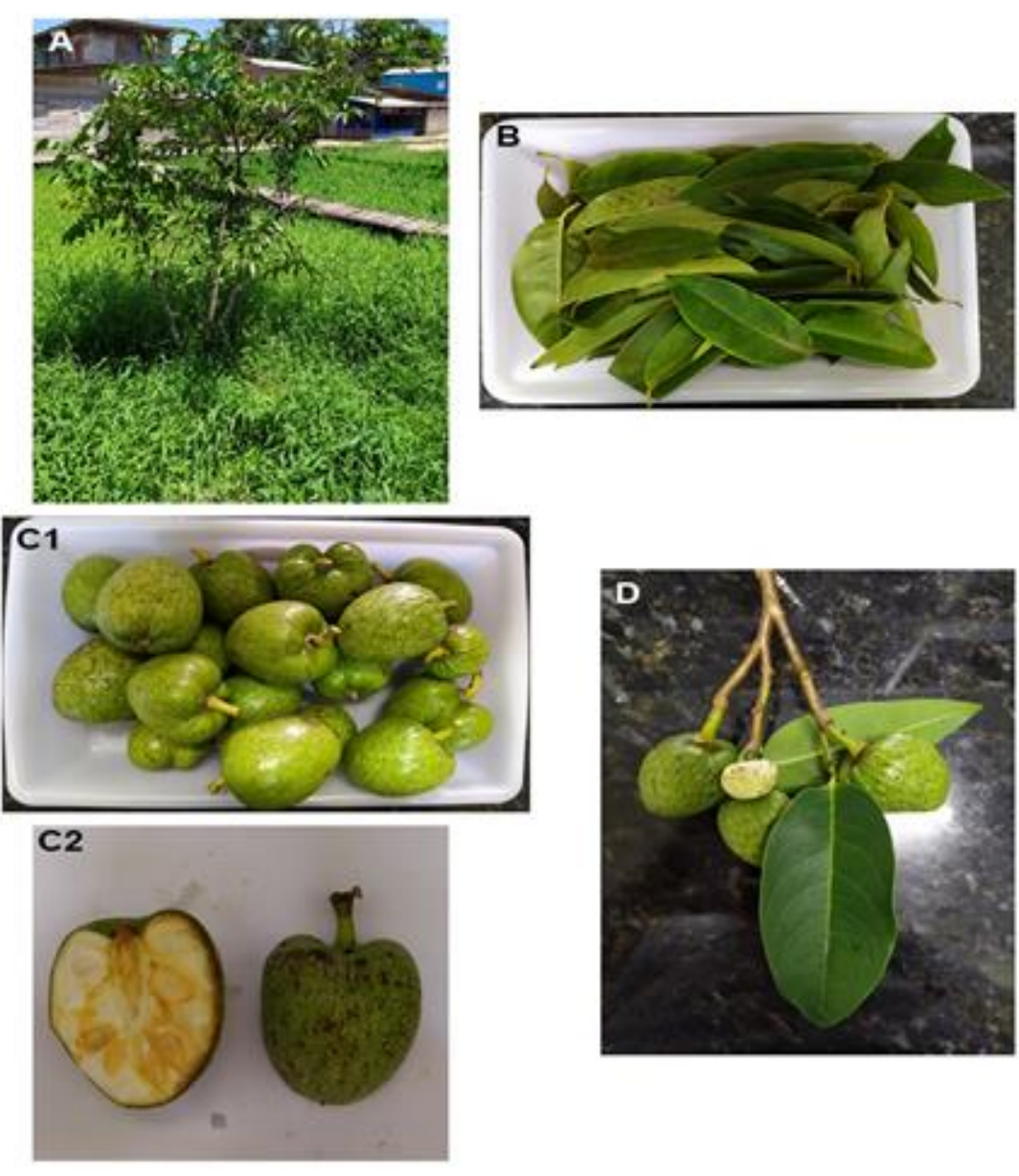

Figura 1. Annona glabra L. (A); Folhas (B); Frutos (C1 e C2); e Ramo floral - folhas, flor em desenvolvimento e frutos (D).

\section{Marco teórico}

A família Annonaceae é o grupo de maior relevância inserido na ordem Magnoliades, constituído aproximadamente de 135 gêneros e 2.500 espécies, sendo considerada uma das maiores entre as angiospermas basais (Chatrou, Rainer, \& Maas, 2004; Group, 2009; APG, 2003; Tsou \& Fu, 2002). O gênero Annona L. nativa da região Neotropical - Américas (Central e Sul) e África tropical, é formado por cerca de 175 espécies (Fries, 1959; Larranaga \& Hormaza, 2015). Os frutos produzidos por algumas espécies do gênero eram apreciados e cultivados por civilizações pré-colombianas na América Central e do Sul (Larranaga \& Hormaza, 2015; NAP, 1989) sendo esta prática mantida até os dias atuais em países em desenvolvimento com climas tropicais e subtropicais (Larranaga \& Hormaza, 2015).

A espécie Annona glabra L. (Annonaceae), Annona australis, Annona palustris ou Annona palustris var. grandiflora, possui diversos nomes populares como araticum do brejo, araticumcortiça, araticum da praia, araticum, araticum de jangada, araticum do rio, araticum-panã, anonalisa, panã, maçã de jacaré, araticupana e ou em inglês pond-apple (Corrêa, 1984; Pontes, Barbosa, \& Maas, 2004). A espécie A. glabra é constituída por plantas de pequeno porte (arbustos ou arvoretas), frutífera, com ampla distribuição geográfica e é encontrada nas margens de lagos e rios, se desenvolvendo em 


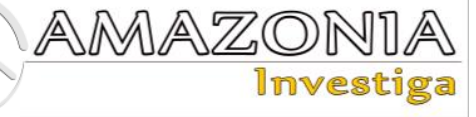

ambientes sujeitos a inundações podendo durar por períodos curtos, longos ou até constantemente inundados (Campbell et al., 1992; Croat, 1978), isso se deve ao seu sistema hidráulico de troncos e galhos eficientes, similar a outras espécies encontradas em regiões tropicais e temperadas (Scherer, 1995; Zotz, 1995; Zotz et al., 1997).

Em território brasileiro, a espécie apresenta uma ampla distribuição passando pelos estados do Amapá, Pará, Paraíba, Maranhão, Ceará, Pernambuco, Bahia, Alagoas, Sergipe, Minas Gerais, Espírito Santo, São Paulo, Rio de Janeiro, Paraná e Santa Catarina (Lobão, Araujo, \& Kurtz, 2005; Maas et al., 2001; Pontes et al., 2004).

As plantas dessa espécie (Figura 1) possuem folhas oblongas ou ovado-lanceoladas, cartáceas, subcoriáceas e glabras com pecíolo longo; flores isoladas, terminais, com pétalas carnudas, coloração esbranquiçada típica das Annonaceae tropicais e com uma fragrância que remete à acetona; os frutos são de tom amarelado, sincárpico e obovado à ovado semelhante a uma maçã, contudo o consumo não se destaca frente a outros representantes do gênero (CNCFlora, 2012; Chatrou et al., 2012; Chen, Chang, Cho \& Wu, 2000; Group, 2009; Larranaga \& Hormaza, 2015; Lobão et al., 2005; Maas et al., 2001; Matsumoto et al., 2014; Pontes et al., 2004).

Sua madeira apresenta diversas virtudes aumentando seu valor comercial com aplicabilidades em torno da carpintaria, caixotaria, confecções de ripas, mastros e remos de pequenas embarcações e suas raízes têm como finalidade a confecção de cortiças (Lobão et al., 2005).
O período de floração, no estado do Rio de Janeiro, ocorre em junho, setembro, novembro e dezembro (Lobão et al., 2005) e a frutificação passa pelos meses de janeiro a março, junho e dezembro, com a dispersão dos frutos ocorrendo por hidrocoria (CNCFlora, 2012). Através do trabalho de Goodrich \& Raguso (2009), é sugerido que besouros realizam a polinização da espécie devido a característica do odor e por se intensificar após o pôr do sol (Gottsberger, 1999; Jürgens, Webber \& Gottsberger, 2000; Silberbauer-Gottsberger, Gottsberger \& Webber, 2003).

Devido a sua biologia, a espécie se torna ideal para estudos de adaptações fisiológicas de plantas tolerantes a ambientes ricos em recursos hídricos, além de exercer um importante papel na sucessão ecológica, como fica evidenciado em alguns estudos (Croat, 1978; Zotz et al., 1997).

\section{Metodologia}

Os artigos selecionados para o trabalho forneceram dados sobre a aplicabilidade da espécie A. glabra na área da saúde, composição química e atividade biológica (Figura 2), sendo desconsiderados os artigos que não preencheram esses requisitos. A seleção dos trabalhos ocorreu através da utilização de palavras chaves em diferentes combinações: Annona glabra, saúde, compostos químicos e atividade biológica. Plataformas bibliográficas utilizadas: Annual Reviews, Europe PMC, Google Acadêmico, Indian Journals, PubMed, ResearchGate, SAGE journals, Scielo, ScienceDirect, Scientific Reports, Semantic Scholar, Wiley Online Library e J-STAGE. 


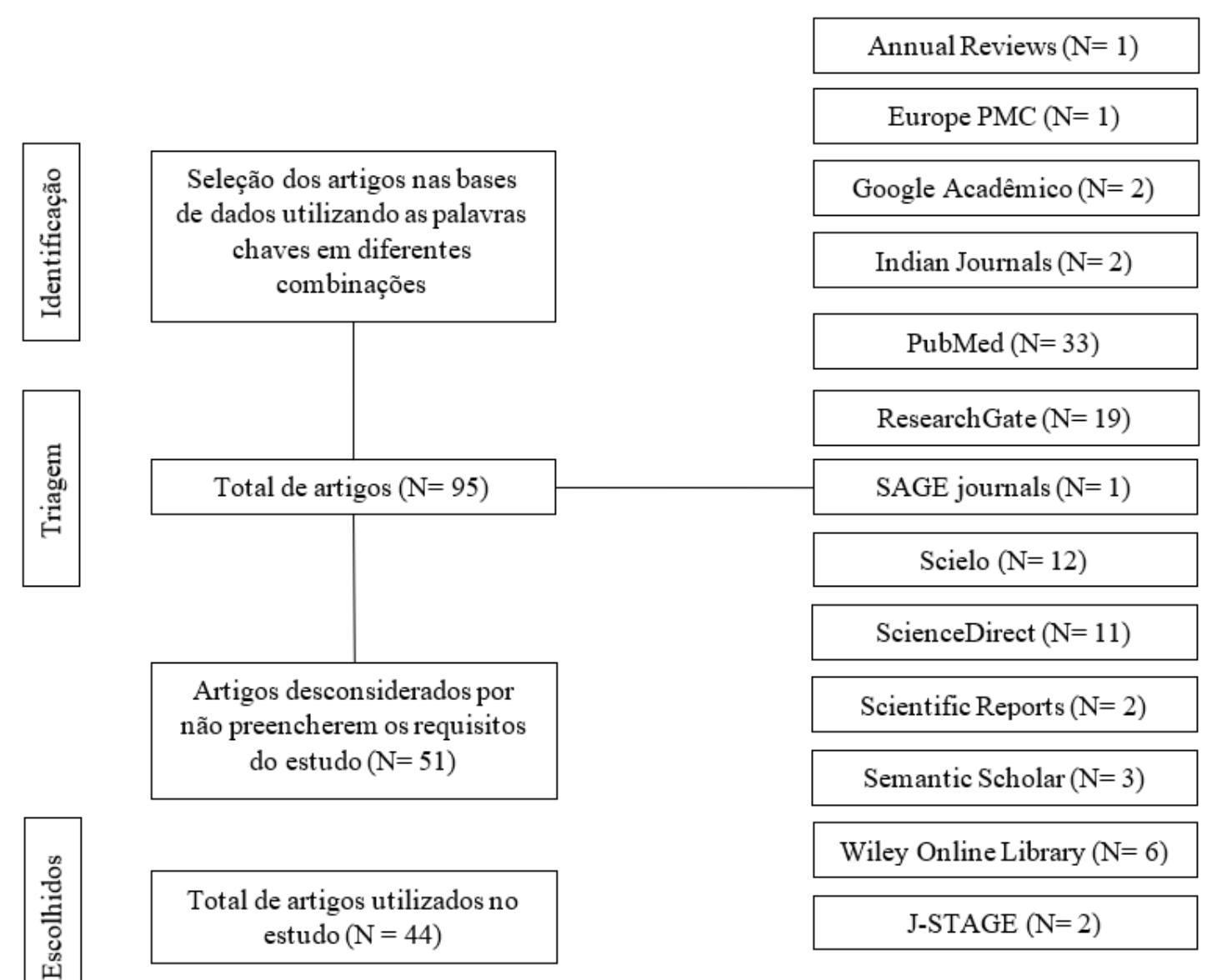

Figura 2. Diagrama de fluxo para seleção dos artigos.

\section{Resultados e discussões}

Os artigos que compõem este estudo analisaram os constituintes químicos das diferentes partes da planta A. glabra, sua atividade biológica e ação farmacológica (Tabela 1). Diversas linhas de pesquisas se debruçaram sob os constituintes químicos, a fim de entender melhor a composição particular de cada estrutura da espécie A. glabra e sua possível aplicabilidade ligada a área da saúde.

As acetogeninas anonáceas são derivadas de ácidos graxos de cadeia longa, sendo os constituintes majoritários nos membros da família Annonaceae (Alali, Liu, \& McLaughlin, 1999). Elas formam uma classe de compostos com propriedades anticarcinogênica, capazes de inibir a NADH oxidase da membrana plasmática ligada à ubiquinona das células cancerosas e a produção de ATP via bloqueio do complexo I mitocondrial (Cochrane et al., 2008; Gallardo et al., 1998; Liu et al., 1998; Miyoshi et al., 1998; Morré et al., 1995); apresentam atividade antimalárica (Balbach, 1995); antiparasitária
(Balbach, 1995; Ohsawa et al., 1991); e inseticida, interferindo no transporte de elétrons nas mitocôndrias dos insetos (de Mendonça et al., 2005 Ohsawa et al., 1991; Padmaja et al., 1995; Zeng et al., 1996).

Ao trabalhar com extrato metanólico do caule de A. glabra (Chen et al., 2000) descobriram três novos diterpenóides de caurano: anoglabasina $\mathrm{C}$ (16 $\alpha$-acetoxi-ent-cauran-19-óico ácido-17-éster metílico) anoglabasina D (éster $16 \alpha$-acetoxi-entcauran-19-al-17-metil) e anoglabasina E (ácido 16 $\alpha$-hidro-19-ol-ent-cauran-17-óico); um novo diterpenóide norcaurano, anoglabasina F (16 $\alpha$-acetoxi-19-nor-ent-cauran-4 $\alpha$-ol-17metil éster) e 13 cauranos derivados conhecidos (ácido 16 $\alpha$-Metoxi-ent-cauran-19-óico, éster 16 $\alpha$-hidro-ent-cauran-17,19-dimetil, ácido 16 $\beta$,17-dihidroxi-ent-cauran-19-óico, ácido $16 \beta$,17-diacetoxi-ent-cauran-19-óico, ácido $16 \alpha-$ hidro-ent-cauran-17,19-dióico, ácido ent-caur16-en-19-óico, ácido 16 $\alpha$-hidro-19-al-entcauran-17-óico, éster 16 $\alpha$-hidro-19-al-entcauran-17-metil, ácido 16 $\alpha$-hidroxi-ent-cauran19-óico, ácido 16 $\alpha$-hidro-ent-cauran-17-óico, 


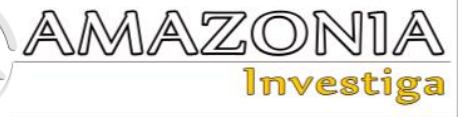

ácido ent-caur-15-en-17-ol-19-óico, ent-caur-15en-17,19-diol e ácido 19-nor-ent-cauran-4 $\alpha$-ol17-óico). Pela primeira vez foram isolados a partir de fonte natural o ácido $16 \alpha$-Metoxi-entcauran-19-óico e o éster 16 $\alpha$-hidro-ent-cauran17,19-dimetil. Já no estudo realizado por (Chen et al., 2004), descobriram um novo diterpenóide dimérico de caurano denominado annoglabayin nos frutos de A. glabra.

$\mathrm{Na}$ busca por bioativos, utilizando extratos etanólicos das folhas de A. glabra, foram isolados os compostos glacinas A e B. Essas acetogeninas foram submetidas a testes de citotoxidade in vitro contra diferentes linhagens celulares tumorais, sendo demonstrada sua capacidade e seletividade (Liu et al., 1998). Em seus trabalhos Matsumoto et al. (2014) e Rocha et al. (2017), isolaram e identificaram a partir das folhas, oito compostos, sendo dois esteróides ( $\beta$-sistosterol e estigmasterol), cinco diterpenos com o esqueleto de caurano (ácido ent-caur-16en-19-óico, ácido ent-19-metoxi-19-oxocauran17-óico, anoglabasina $\mathrm{B}$, ácido ent-17hidroxicaur-15-en-19-óico e ácido ent-15 $\beta, 16 \beta$ epoxi-17-hidroxi-cauran-19-óico) e a acetogenina asimicina. Já Lee et al. (2015), através dos extratos etanólicos provenientes das folhas, obteve os seguintes compostos: (-)- $N$ metilactinodafinina, (-)-actinodafinina, $(+)$ boldina, (-)-anolobina, (-)-asimilobina, $(+)$-norisodomesticina, (-)-roemerolina, (+)reticulina, (-)-(6aS,7R)-7-hidroxiactinodafinina, (+)-1S,2S-reticulina $N$-óxido, liriodenina, (-)palidina, $(+)$-estearina, $(+)$-magnofloro, quercetina, quercetina-3-O- $\beta$-Dgalactopiranosídeo, 3-O- $\beta$-D-glucopiranosídeo e 3-O- $\alpha$-L-arabinopiranosídeo. Entre eles, (-) anolobina e (-) - roemerolina demonstravam atividade inibitória satisfatória contra acetilcolinesterase com valores de $\mathrm{IC}_{50}$ (concentração inibitória) de 22,4 e 26,3 $\mu \mathrm{M}$, respectivamente. A hipótese colinérgica levantada pelos autores seria uma alternativa para o tratamento do Alzheimer, onde esses inibidores atuariam sobre a taxa de acetilcolina nas sinapses colinérgicas do córtex cerebral (Lee et al. 2015).

Chang et al. (1998) descobriram dois novos diterpenóides cauranos derivados dos frutos, anoglabasina A (metil-16 $\beta$-acetoxi-19-al-entcauran-17-oato) e anoglabasina B (16 $\alpha$-hidro-19acetoxi-ent-ácido cauran-17-óico). Outros compostos foram encontrados, porém estes já haviam sido isolados anteriormente: ácido entcaur-16-en-19-óico; ácido 16 $\alpha, 17$-dihidroxi-entcauran-19-óico; ácido 16 $\beta$-hidroxi-17-acetoxient-cauran-19-óico; ácido 16 $\beta$-hidro-ent-cauran- 17-óico; ácido 16 $\alpha$-hidro-ent-cauran-17-óico; ent-caur-16-en-19-ol; $\quad$ ent-caur-15-eno-17,19diol; ácido 16 $\alpha$-hidro-19-al-ent-cauran-17-óico; metil-16 $\alpha$-hidro-19-al-ent-cauran-17-oato; $16 \beta$ hidroxil-17-acetoxy-ent-cauran-19-al e ácido 19nor-ent-cauran- $4 \alpha$-ol-17-oico. Contudo verificou-se que o composto metil-16 $\alpha$-hidro-19al-ent-cauran-17-oato demonstrou atividade leve contra a replicação do HIV em células de linfócitos H9 e o ácido 16 $\alpha$-17-dihidroxi-entcauran-19-óico inibiu significativamente a transcriptase reversa do HIV.

A obtenção dos compostos derivados do material vegetal pode variar de acordo com a técnica que será empregue e o tipo de amostra a ser analisada durante o trabalho. Ao estudarem a composição química floral de A. glabra, foram encontrados os compostos 1,8-cineol (eucaliptol), acetato de 3-pentanil e 3-pentanol, produtos esses majoritários desta estrutura (Goodrich \& Raguso, 2009). Li et al. (1998 e 1999), trabalhando apenas com as sementes isolaram e caracterizaram os compostos glabrina A, B, C e D.

Chang et al. (2000) isolaram uma série de compostos a partir dos frutos e caules, sendo dioxoaporfina - annobraine; duas oxoaporfinas liriodenina e lisicamina; cinco aporfinas - (-)nornuciferina,

$(-)$-anonaina, (-)- $N$-formilanonaine, (-)-asimilobina e (+)nordomesticina; uma proaporfina (+)-esteparina; duas protoberberinas - (-)quicemanina e desidrocorydalmina; uma azaantraquinona - 1-aza-4-metil-2-oxo-1,2dihidro-9,10-antracenodi-ona; duas amidas $-N$ trans-feruloiltiramina e $N$ - $p$-coumaroiltiramina; uma ionona - blumenol A; cinco esteróides - $\beta$ sitosterol, estigmasterol, $B$-sitosteril-Dglucosídeo, estigmasteril-D-glucosídeo e 6-Opalmitoil-ß-sitosteril-D-glucosídeo.

Em trabalho realizado com Annona spp. provenientes do Vietnã, foi analisada a composição química dos diferentes óleos voláteis. Entre as espécies a A. glabra apresentou como constituintes majoritários $\beta$-cariofileno $(21,5 \%)$, germacreno D $(17,7 \%), \alpha$-cadinol (5,4\%), $\beta$-elemeno $(5,2 \%), \alpha$-felandreno $(4,3 \%)$ e $\alpha$-cariofileno (3,6\%) (Thang, Dai, Hoi, \& Ogunwande, 2013). Foi observado variações na composição e nas concentrações dos compostos isolados das suas amostras em relação a outros autores, como visto no trabalho de Santos et al. (1998), que analisaram os óleos dos frutos de A. glabra no Brasil em três diferentes estágios (verde, meio maduro e maduro) - terpenóides $\alpha$ pineno $(18,5 ; 11,3 ; 15 \%)$, limoneno $(20 ; 20,7$; $20,6 \%), \alpha$-felandreno $(21 ; 1,2 ; 2 \%)$ e $(E)-\beta$ - 
ocimeno $(15,8 ; 19,7 ; 18 ; 3 \%)$. Enquanto que Pino; Marbot; Agüero (2002) com bagas provindas de Cuba, encontraram mirceno (47,1\%), (Z)- $\beta$-ocimeno (16,3\%), limoneno $(11,2 \%)$ e $\alpha$-pineno $(9,5 \%)$, sendo setenta e oito compostos identificados pela primeira vez como componentes voláteis nesta planta. Os sesquiterpenos foi a classe dominante do óleo de A. glabra para a região do Vietnã, divergindo do que foi observado com os óleos do Brasil e de Cuba com os terpenóides (Pino, Marbot, \& Agüero, 2002; Santos et al., 1998). A diferença nos constituintes químicos dos óleos de uma planta pode variar de acordo com inúmeros fatores bióticos (parte analisada, idade da planta, presença de predadores e parasitas e o local de coleta das amostras) e abióticos (estação climática, técnica de coleta, nutrientes disponíveis, temperatura e luz).

Através do trabalho de Anh et al. (2014), foi descoberto um novo glicosídeo ent-caurano (anoglabasina $\mathrm{H})$ e isolados três compostos já conhecidos anoglabasina $\mathrm{E}$, anoglabasina B e ácido 19-nor-ent-caurent-4-ol-17-óico, a partir dos frutos de A. glabra coletados na cidade de Ho Chi Minh (Vietnã). As atividades citotóxicas dos compostos foram aferidas através do ensaio de brometo de 3-[4,5-dimetiltiazol-2-il] -2,5difeniltetrazólio (MTT) sobre quatro linhagens de células de câncer humano (LU-1, MCF-7, SKMel2 e KB), tendo se destacado o composto anoglabasina $\mathrm{H}$, devido sua atividade moderada comprovada em todos os testes com $\mathrm{CL}_{50}$ (concentração letal) entre 3,7 a 4,6 $\mu \mathrm{M}$.

Ao trabalhar como o extrato metanólico dos frutos de A. glabra, Hien et al. (2015) isolaram acetogeninas, ent-cauranos, peptídeos e alcalóides, totalizando nove compostos: um novo ( $2 E, 4 E, 1^{\prime} R, 3^{\prime} S, 5^{\prime} R, 6$ ' $S$ )- ácido di-hidrofásico 1,3 '-di- $O$ - $\beta$-D-glucopiranosídeo e outros oitos compostos conhecidos $\left(2 E, 4 E, 1^{\prime} R, 3^{\prime} S, 5^{\prime} R, 6^{\prime} S\right)$ ácido di-hidrofásico 3'- $O$ - $\beta$-D-glucopiranosídeo, icarisídeo $\quad \mathrm{D}_{2}, \quad$ icarisídeo $\quad \mathrm{D}_{2} \quad 6^{\prime}-O-\beta-\mathrm{D}-$ xilopiranosídeo, 3,4-dimetoxifenil $O-\beta$-Dglucopiranosídeo, ácido 3,4-di-hidroxibenzoico, blumenol A, cucumegastigmane I e icarisida $\mathrm{B}_{1}$. $\mathrm{O}$ composto icarisídeo $\mathrm{D}_{2}$ apresentou atividade citotóxica (teste MTT) significativa na linhagem celular de câncer HL-60 com IC $_{50}$ de 9,0 \pm 1,0 $\mu \mathrm{M}$, sendo observado alterações nas proteínas relacionadas a apoptose, sinalizando alteração na expressão das mesmas e diminuição da fosforilação de AKT em células HL-60.

As partes aéreas (galhos) da espécie A. glabra são fontes de substâncias com função inseticida (Chen et al., 2000; de Mendonça et al., 2005;
Ohsawa et al., 1991; Padmaja et al., 1995; Zeng et al., 1996), esporicida (Padmaja et al., 1995), atividade citotóxica e ação larvicida sob a espécie A. aegypti (Amarasinghe et al., 2020; de Mendonça et al., 2005). Em estudos preliminares, o extrato hexânico da casca do caule apresentou ação antimicrobiana e antihelmíntica, tendo como constituinte majoritário o ácido (-) caur-16-en-19-óico (Padmaja et al., 1995).

Nas folhas de A. glabra são encontradas acetogeninas, compostos com atividade contra diferentes células tumorais (Liu, Pilarinou, \& McLaughlin, 1999). De acordo com Padmaja et al. (1995), a casca e folhas são usadas tradicionalmente como inseticida e parasiticida na região de Kerala (Índia). Rocha et al. (2017) analisaram os efeitos dos extratos de acetona provenientes das folhas sobre as algas Rhaphidocelis subcapitata (Korshikov) e no oomiceto Pythium aphanidermatum (Edson). Dentre os resultados obtidos não foi detectado nenhum efeito das concentrações do extrato da folha até $75 \mathrm{mg} / \mathrm{L}$, porém efeitos inibitórios iniciaram a partir de $125 \mathrm{mg} / \mathrm{L}$ em $R$. subcapitata. Os efeitos foram a redução da taxa de crescimento e da biomassa final das algas, tendo sido alcançado inibição total das microalgas a partir da concentração de $200 \mathrm{mg} / \mathrm{L}$, sem haver diferença estatística significativa entre as três maiores concentrações. Os autores destacam que os efeitos inibitórios sobre $P$. aphanidermatum na concentração de 125 $\mu \mathrm{g} /$ disco foram similares aos fungicidas Cicloheximida e Bifonazol. Pressupõem que tais resultados se devam aos constituintes químicos das folhas da planta, pois estes são apontados pela literatura possuindo atividade antifúngica, o que explicaria também a atividade anti-oomiceto.

Zhang et al. (2004) estudaram os efeitos inibitórios de dois diterpenóides, ácido cunábico (ácido ent-caur-16-en-19-óico) e o ácido entcauran-19-al-17-óico, derivados de A. glabra na proliferação celular de câncer de fígado humano (CFH) SMMC-7721 através do ensaio MTT. Os resultados levantados pela pesquisa mostram efeito inibitório para ambos diterpenóides, apresentando dose dependente por tempo, onde a dosagem mínima necessária foi de $>5 \mu \mathrm{mol} / \mathrm{L}$ para ácido cunábico e de $>10 \mu \mathrm{mol} / \mathrm{L}$ para ácido ent-cauran-19-al-17-óico. $\mathrm{O}$ maior efeito inibitório de crescimento registrado envolveu o ácido cunábico $(81,05 \%$ a $25 \mu \mathrm{mol} / \mathrm{L}, 72 \mathrm{~h})$, porém em dosagens mais elevadas a inibição não seguiu essa tendência. Para o ácido ent-cauran19-al-17-óico, o maior percentual inibitório foi de $56,86 \%$ a $75 \mu \mathrm{mol} / \mathrm{L}$ (72h), sendo observado 


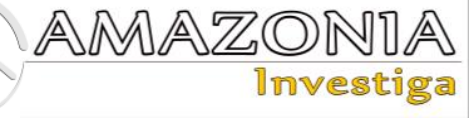

neste caso o efeito inibitório dependente da concentração e do tempo. Alterações morfológicas foram observadas com auxílio da microscopia de contraste de fase, fluorescencia, eletrônica de transmissão e de varredura. As anomalias encontradas nos testes envolviam apoptose, cromatina condensada e redução no volume. Através do citometria de fluxo foi obtida a porcentagem apoptótica celular, onde o ácido cunábico e ácido ent-cauran-19-al-17-óico (25 $\mu \mathrm{mol} / \mathrm{L}$ a $48 \mathrm{~h})$ registraram $43,31 \%$ e $24,95 \%$, respectivamente. Os compostos interferiram no ciclo celular bloqueando a progressão das células SMMC-7721 durante a fase $\mathrm{S}$ causando parada no estágio $\mathrm{G}_{0} / \mathrm{G}_{1}$. Análises imunohistoquímicas revelaram que houve indução de apoptose por meio da regulação negativa da expressão do gene $b c l-2$ e pela regulação positiva do gene bax. Os autores concluíram que os dois compostos diterpenóides atuaram inibindo de forma satisfatória na proliferação das células CFH SMMC-7721.

Através do trabalho de Galvão et al. (2016), foi demonstrado que os flavonóides derivados da fração de acetato de etila (FAE) das folhas de A. glabra possuem propriedades antimicrobianas sob Pseudomonas aeruginosa. A atividade antibacteriana ocorre em decorrência da atuação sob a superfície da parede celular bacteriana. Os resultados obtidos com FAE mostram atividade bacteriana contra diferentes cepas de $P$. aeruginosa e a sub-fração que mais se destacou foi SF32-33, abundante em flavonóides. Segundo Fang et al. (2016), os flavonóides interferem na viabilidade de Escherichia coli em decorrência de danos provocados na sua membrana externa. Wu et al. (2013) observaram a interação dos flavonóides com proteínas da parede celular de E. coli, acarretando na alteração de sua estrutura. Tal efeito poderia ter se repetido em $P$. aeruginosa quando submetida a SF 32-33 interferindo na sua viabilidade e crescimento. A SF32-33 apresentou os seguintes flavonóides: (-) epicatequina, fisetina, quercetina, rutina (glicosídeo de quercetina), hiperosídeo, isoquercitrina, quercitrina, caempferol 7-neohesperidosídeo, nicotiflorina e naringenina. Os resultados demonstram a viabilidade do uso do FAE das folhas de A. glabra no tratamento de infecções oriundas de $P$. aeruginosa (Galvão et al., 2016).

A eficácia anticarcinogênica dos extratos alcoólicos das folhas A. glabra foi estudada sobre as células de leucemia monocítica humana (LIU et al., 2018). As análises realizadas indicaram a presença de flavonóides, terpenóides, glicosídeos, esteróides, saponinas, taninos, antraquinonas e compostos ácidos nos extratos. Foram testadas 5 concentrações (20 a 100 $\mu \mathrm{g} / \mathrm{mL}$ ) tendo sido observada uma redução na viabilidade das células leucêmicas (1,8 a 27,3\%) exibindo uma relação dose-dependencia; alterações morfológicas como mitocôndrias condensadas, fragmentadas e aglomeradas foram registradas; ocorrência de apoptose nas concentrações $80 \mu \mathrm{g} / \mathrm{mL}(10,7 \%)$ e $100 \mu \mathrm{g} / \mathrm{mL}$ $(22,4 \%)$ dos extratos; e redução na migração das células leucêmicas. Segundo os autores, os extratos das folhas figuram como potenciais agentes anticâncer contra células de leucemia humana. Os ensaios realizados ABTS, DPPH e FRAP confirmaram a capacidade de eliminar os radicais livres por parte dos extratos de folhas de A. glabra. Com base nos dados obtidos, foi possível atestar a eficácia in vitro do extrato foliar de A. glabra em células de leucemia humana (LIU et al., 2018).

Amarasinghe et al. (2020) analisaram o potencial larvicida de nanopartículas derivadas das folhas de A. glabra (An-AgNPs) e o extrato aquoso bruto no controle de larvas (L3) de A. aegypti e Aedes albopictus. Os resultados com An-AgNPs foram considerados satisfatórios com $\mathrm{CL}_{50}-24 \mathrm{~h}$ de exposição: A. aegypti (extrato vegetal: $\mathrm{AgNO}_{3}$ 1:10) $5,29 \mathrm{mg} / \mathrm{L}$ e $\left(\mathrm{AgNO}_{3} 2: 10\right) \quad 2,43 \mathrm{mg} / \mathrm{L}$; enquanto A. albopictus $\left(\mathrm{AgNO}_{3} 1: 10\right) 3,02 \mathrm{mg} / \mathrm{L}$ e $\left(\mathrm{AgNO}_{3} 2: 10\right) 2,51 \mathrm{mg} / \mathrm{L}$. Os valores de $\mathrm{CL}_{50}$ (24h) obtidos para o extrato aquoso bruto contra A. aegypti e A. albopictus foram 5,94 mg/L e 5,00 $\mathrm{mg} / \mathrm{L}$, respectivamente. A espécie A. albopictus demonstrou ser mais suscetível em ambos os testes (An-AgNPs e extrato aquoso bruto), com exceção do teste An-AgNPs $\left(\mathrm{AgNO}_{3} 2: 10\right)$ e entre os produtos analisados as nanoformulações demonstraram atividade larvicida superior no controle dos mosquitos vetores.

O estudo realizado por de Mendonça et al. (2005) testou uma série de extratos e óleos de plantas medicinais brasileiras a fim de aferir as atividades inseticidas, utilizando a metodologia preconizada pela OMS (WHO, 1996). O extrato etanólico mais ativo foi de A. glabra, preparado em aparelho Soxhlet a partir do caule, apresentando uma $\mathrm{CL}_{50}$ de $27 \mu \mathrm{g} / \mathrm{L}$ (de Mendonça et al., 2005). Os resultados encontrados para as larvas de A. aegypti não são inesperados, pois Mendonça (2003) já havia demonstrado a atividade inseticida da planta, desta vez, derivada dos extratos das sementes.

dos Santos \& Sant'Ana (2001), realizaram testes para avaliar a atividade dos extratos etanólicos de seis espécies de Annonaceae, entre elas encontrava-se A. glabra. A maioria dos extratos 
analisados sobre Biomphalaria glabrata, massa de ovos e fase adulta, apresentou letalidade sobre o molusco. B. glabrata possui papel vital no ciclo de vida da esquistossomose servindo como hospedeiro intermediário e o controle desse invertebrado é fundamental para reduzir o número de casos da doença. Os testes para ambas as fases de desenvolvimento do caracol seguiram o modelo preconizado pela OMS (WHO, 1983). Os resultados encontrados para os extratos derivados das folhas e sementes (ppm) de A. glabra sobre $B$. glabrata (fase adulta) foram $\operatorname{DL}_{50}(0,97$ e 9,23$)$ e $\operatorname{DL}_{90}(3,79$ e 17,02$)$, contudo para a massa de ovos foi observada inatividade dos extratos Santos \& Sant'Ana (2001).

Tabela 1.

Constituintes químicos encontrados em diferentes partes de Annona glabra.

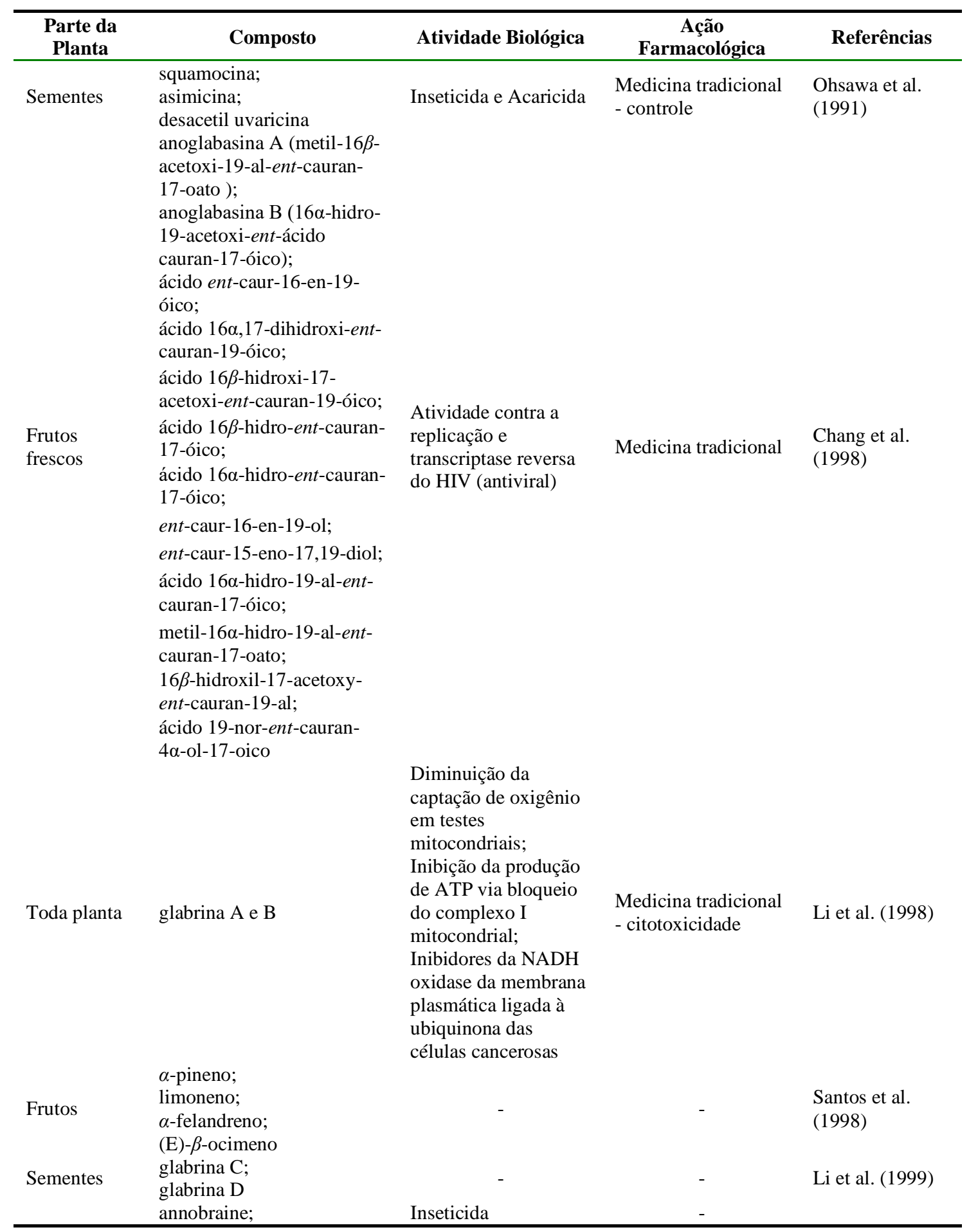




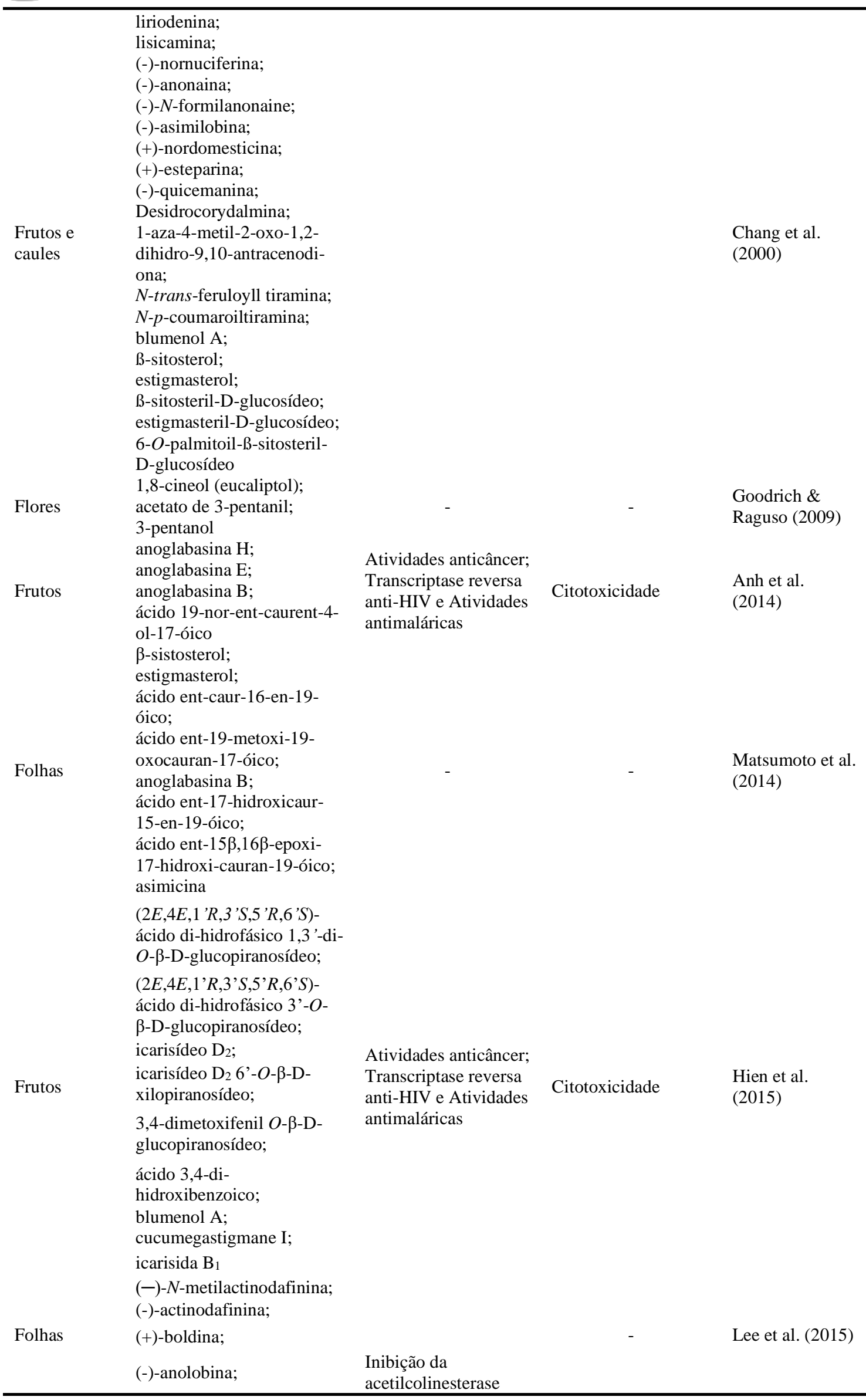




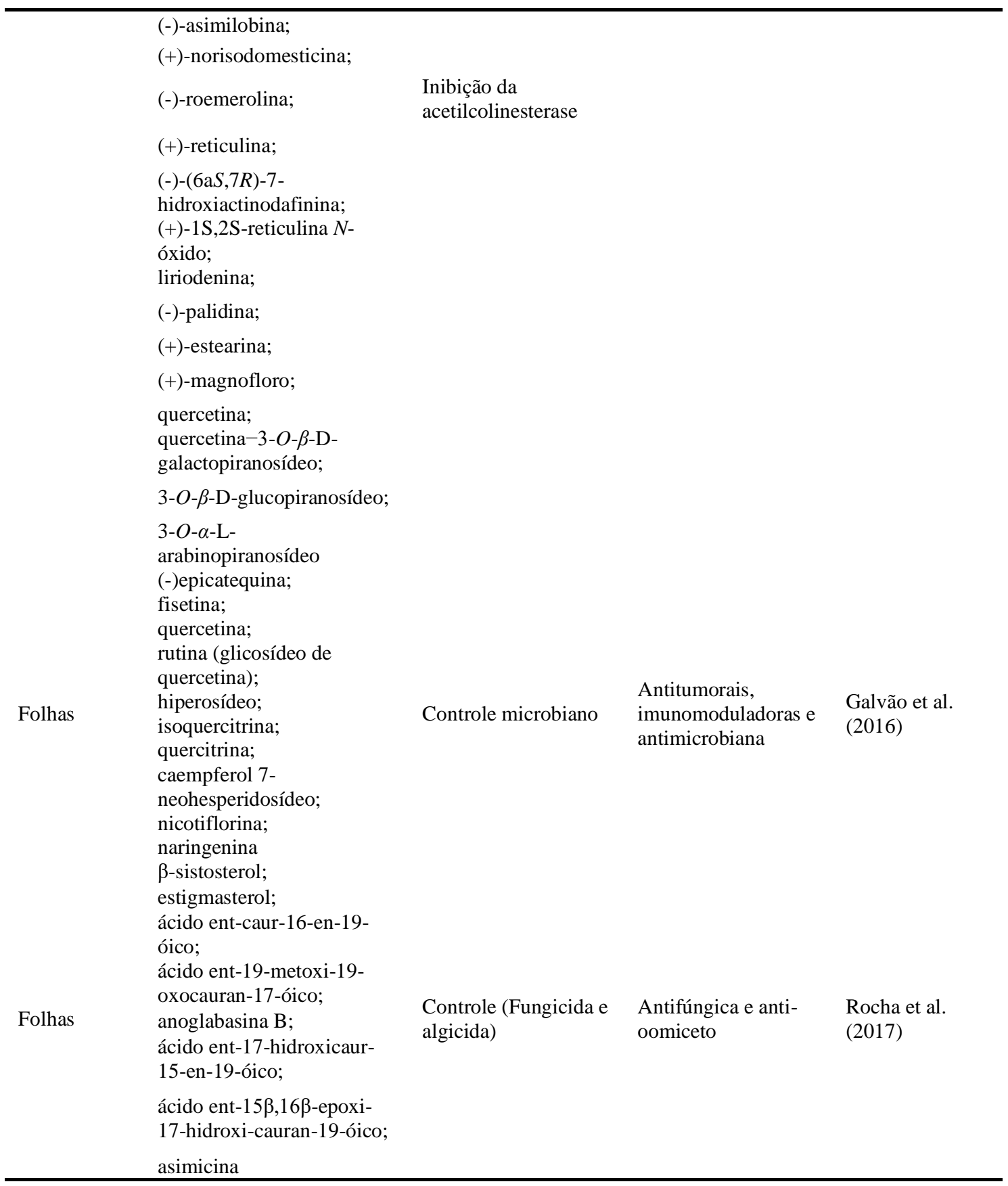

\section{Conclusões}

Os estudos sobre a espécie A. glabra apresentam dados promissores sobre atividades anticarcinogênica, antioxidantes, antirreumática, antimicrobiana, antifúngica, inseticida, vermicida e antiparasitária. Apesar de ser utilizada a tempos na medicina tradicional, infelizmente as informações acerca de suas atividades alelopáticas são pouco conhecidas devido à escassez de pesquisas e consequentemente nenhum estudo alcançou a fase de testagem clínica.
Mais estudos sobre sua bioquímica e fisiologia necessitam ser realizados, a fim de atestar essas propriedades. Caso sejam confirmados, testes mais aprofundados e voltados à elaboração de um produto final precisam ser realizados para atender as necessidades humanas. Este estudo buscou ser fonte de estímulo para novas pesquisas abordando a atividade biológica e farmacológica, in vitro e in vivo, das diferentes peças florais de A. glabra.

\section{Referências bibliográficas}

Alali, F. Q., Liu, X. X., \& McLaughlin, J. L. (1999). Annonaceous acetogenins: Recent 


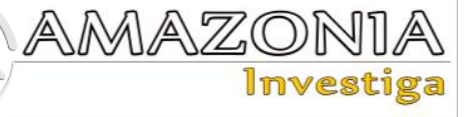

progress. Journal of Natural Products, 62(3), 504-540. https://doi.org/10.1021/np980406d.

Amarasinghe, L. D., Wickramarachchi, P. A. S., Aberathna, A. A. A. U., Sithara, W. S., \& De Silva, C. R. (2020). Comparative study on larvicidal activity of green synthesized silver nanoparticles and Annona glabra (Annonaceae) aqueous extract to control Aedes aegypti and Aedes albopictus (Diptera: Culicidae). Heliyon, 6(6). https://doi.org/10.1016/j.heliyon.2020.e0432 2.

Anh, H. L. T., Hien, N. T. T., Hang, D. T. T., Ha, T. M., Nhiem, N. X., Hien, T. T. T., Kiem, P. V. (2014). Ent-Kaurane diterpenes from Annona glabra and their cytotoxic activities. Natural Product Communications, 9(12), 1681-1682.

Balbach, A. (1995). Book: Plants Heal (1ª). São Paulo, Brazil: Full life. ISBN 19951410.

Campbell, D. G., Stone, J. L., \& Rosas, A. (1992). A comparison of the phytosociology and dynamics of three floodplain (Várzea) forests of known ages, Rio Juruá, western Brazilian Amazon. Botanical Journal of the Linnean Society, 108(3), 213-237. https://doi.org/10.1111/j.10958339.1992.tb00240.x.

CNCFlora. (2012). Annona glabra in Red List of Brazilian flora version 2012.2 National Center for the Conservation of Flora, November 22, 2020. http://cncflora.jbrj.gov.br/portal/ptbr/profile/Annona\%20glabra.

Chang, F. R., Yang, P. Y., Lin, J. Y., Lee, K. H., \& Wu, Y. C. (1998). Bioactive kaurane diterpenoids from Annona glabra. Journal of Natural Products, 61(4), 437-439. https://doi.org/10.1021/np970497z.

Chang, F. R., Chen, C. Y., Hsieh, T. J., Cho, C. P., \& Wu, Y. C. (2000). Chemical Constituents from Annona Glabra III. Journal of the Chinese Chemical Society, 47(4B), 913-920. https://doi.org/10.1002/jccs.200000124

Chatrou, L. W., Rainer, H., \& Maas, P. J. M. (2004). Flowering Plants of the Neotropics. Princeton University Press. New York: Smith, N. et al. ISBN 978-0-691-11694-5.

Chatrou, Lars W., Pirie, M. D., Erkens, R. H. J., Couvreur, T. L. P., Neubig, K. M., Abbott, J. R., Chase, M. W. (2012). A new subfamilial and tribal classification of the pantropical flowering plant family Annonaceae informed by molecular phylogenetics. Botanical Journal of the Linnean Society, 169(1), 5-40. https://doi.org/10.1111/j.1095-

8339.2012.01235.x.

Chen, C. Y., Chang, F. R., Cho, C. P., \& Wu, Y. C. (2000). Ent-kaurane diterpenoids from Annona glabra. Journal of Natural Products, 63(7), 1000-1003. https://doi.org/10.1021/np0000320.

Chen, C. H., Hsieh, T. J., Liu, T. Z., Chern, C. L., Hsieh, P. Y., \& Chen, C. Y. (2004). Annoglabayin, a novel dimeric kaurane diterpenoid, and apoptosis in Hep G2 cells of annomontacin from the fruits of Annona glabra. Journal of Natural Products, 67(11), 1942-1946. https://doi.org/10.1021/np040078j.

Chil Núñez, I., Escalona Arranz, J., Molina Bertrán, S., Dutok Sánchez, C., Arias Ramos, D., Pérez Rondón, L., Ochoa Pacheco, A., \& Picanço Souto, R. (2020). Perfeccionamiento de la estrategia curricular de medio ambiente de la carrera de ciencias farmacéuticas de la Universidad de Oriente, Cuba. Revista Científica Del Amazonas, 3(5), 6-17. https://doi.org/10.34069/RC/2020.5.01

Cochrane, C. B., Nair, P. K. R., Melnick, S. J., Resek, A. P., \& Ramachandran, C. (2008). Anticancer effects of Annona glabra plant extracts in human leukemia cell lines. Anticancer Research, 28(2A), 965-971.

Corrêa, M. P. (1984). Dictionary of Useful Plants of Brazil and Cultivated Exotic Plants. Ministry of Agriculture, Brazilian Institute for Forestry Development (Vols. 1-1, 2 and 3). Brazil. ISBN 198414430.

Croat, T. B. (1978). Flora of Barro Colorado Island. Stanford University Press.

de Mendonça, F. A. C., da Silva, K. F. S., dos Santos, K. K., Ribeiro J., K. A. L., \& Sant'Ana, A. E. G. (2005). Activities of some Brazilian plants against larvae of the mosquito Aedes aegypti. Fitoterapia, 76(7-8), 629-636. https://doi.org/10.1016/j.fitote.2005.06.013.

Gonçalves, A. E. S. S, Lajolo, F. M., \& Genovese, M. I. (2010). Chemical Composition and Antioxidant/Antidiabetic Potential of Brazilian Native Fruits and Commercial Frozen Pulps. Journal of Agricultural and Food Chemistry, 58(8), 4666-4674. https://doi.org/10.1021/jf903875u.

dos Santos, A. F., \& Sant'Ana, A. E. G. (2001). Molluscicidal properties of some species of Annona. Phytomedicine, 8(2), 115-120. https://doi.org/10.1078/0944-7113-00008.

Fang, Y., Lu, Y., Zang, X., Wu, T., Qi, X., Pan, S., \& Xu, X. (2016). 3D-QSAR and docking studies of flavonoids as potent Escherichia 
coli inhibitors. Scientific Reports, 6(1), 23634. https://doi.org/10.1038/srep23634.

Fries, R. E. (1959). The natural plant families together with their genera and more important species, especially the useful plants, founded with the participation of numerous outstanding scholars ( $2^{\circ}$ ed, Vol. 17). Berlin, Germany: Engler, A., \& Prantl, K.

Gallardo, T., Aragón, R., Tormo, José, R., Blázquez, M. A., Zafra-Polo, M. C., \& Cortes, D. (1998). Acetogenins from Annona glabra seeds. Phytochemistry, 47(5), 811-816. https://doi.org/10.1016/S00319422(97)00780-2.

Galvão, S. de S. L., Monteiro, A. de S., Siqueira, E. P., Bomfim, M. R. Q., Dias-Souza, M. V., Ferreira, G. F. Monteiro-Neto, V. (2016). Annona glabra Flavonoids Act As Antimicrobials by Binding to Pseudomonas aeruginosa Cell Walls. Frontiers in Microbiology, https://doi.org/10.3389/fmicb.2016.02053.

Genovese, M. I., Pinto, M. D. S., Gonçalves, A. E. S. S., \& Lajolo, F. M. (2008). Bioactive Compounds and Antioxidant Capacity of Exotic Fruits and Commercial Frozen Pulps from Brazil: Food Science and Technology International, 14(3), 207-214. (Sage UK: London, England). https://doi.org/10.1177/1082013208092151.

Goodrich, K. R., \& Raguso, R. A. (2009). The olfactory component of floral display in Asimina and Deeringothamnus (Annonaceae). The New Phytologist, 183(2), 457-469. https://doi.org/10.1111/j.14698137.2009.02868.x

Gottsberger, G. (1999). Pollination and evolution in neotropical Annonaceae. Plant Species Biology, 14(2), 143-152. https://doi.org/10.1046/j.14421984.1999.00018.x.

Group, T. A. P. (2009). An update of the Angiosperm Phylogeny Group classification for the orders and families of flowering plants: APG III. Botanical Journal of the Linnean Society, 161(2), 105-121. https://doi.org/10.1111/j.10958339.2009.00996.x.

Hien, N. T. T., Nhiem, N. X., Yen, D. T. H., Hang, D. T. T., Tai, B. H., Quang, T. H., Kim, Y. H. (2015). Chemical constituents of the Annona glabra fruit and their cytotoxic activity. Pharmaceutical Biology, 53(11), 1602-1607. https://doi.org/10.3109/13880209.2014.9930 42.

Jürgens, A., Webber, A. C., \& Gottsberger, G. (2000). Floral scent compounds of Amazonian Annonaceae species pollinated by small beetles and thrips. Phytochemistry, 55(6), 551-558.

https://doi.org/10.1016/S0031-

9422(00)00241-7.

Larranaga, N., \& Hormaza, J. I. (2015). DNA barcoding of perennial fruit tree species of agronomic interest in the genus Annona (Annonaceae). Frontiers in Plant Science, 6, 589. https://doi.org/10.3389/fpls.2015.00589.

Lee, S. S., Wu, D. Y., Tsai, S. F., \& Chen, C. K. (2015). Anti-Acetylcholinesterase Alkaloids from Annona glabra Leaf. Natural Product Communications, 10(6), 891-893.

Li C. M., Tan N. H., Mu Q., Zheng H. L., Hao X. J., Liang H. L., \& Zhou J. (1998). Cyclopeptides from the seeds of Annona glabra. Phytochemistry, 47(7), 1293-1296. https://doi.org/10.1016/S00319422(97)00752-8.

Li, C. M., Tan, N. H., Zheng, H. L., Mu, Q., Hao, X. J., He, Y. N., \& Zhou, J. (1999). Cyclopeptides from the seeds of Annona glabra. Phytochemistry, 50(6), 1047-1052. https://doi.org/10.1016/S00319422(98)00631-1.

Liu, X. X., Alali, F. Q., Pilarinou, E., \& McLaughlin, J. L. (1998). Glacins A and B: Two novel bioactive mono-tetrahydrofuran acetogenins from Annona glabra. Journal of Natural Products, 61(5), 620-624. https://doi.org/10.1021/np970563x.

Liu, X. X., Pilarinou, E., \& McLaughlin, J. L. (1999). Two novel acetogenins, annoglaxin and 27-hydroxybullatacin, from Annona glabra. Journal of Natural Products, 62(6), 848-852. https://doi.org/10.1021/np980552j.

Liu, Y., Liu, D., Wan, W., \& Zhang, H. (2018). In vitro mitochondria-mediated anticancer and antiproliferative effects of Annona glabra leaf extract against human leukemia cells. Journal of Photochemistry and Photobiology. B, Biology, 189, 29-35. https://doi.org/10.1016/j.jphotobiol.2018.07. 026.

Lobão, A. Q., Araujo, D. S. D. de, \& Kurtz, B. C. (2005). Annonaceae das restingas Do Estado Do Rio de Janeiro, Brasil. Rodriguésia, 56(87), 85-96. https://doi.org/10.1590/217578602005568706.

Maas, P. J. M., de Kamer, H. M., Junikka, L., de Mello-Silva, R., Rainer, H., Maas, P. J. M., \& Rainer, H. (2001). Annonnaceae from Central-eastern Brazil. Rodriguésia, 52(80), 65-98. https://doi.org/10.1590/217578602001528005.

Matsumoto, S., Varela, R. M., Palma, M., Molinillo, J. M. G., Lima, I. S., Barroso, C. G., \& Macías, F. A. (2014). Bio- 


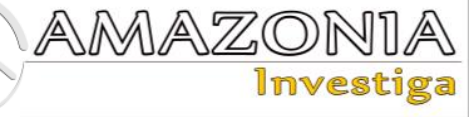

guided optimization of the ultrasoundassisted extraction of compounds from Annona glabra L. leaves using the etiolated wheat coleoptile bioassay. Ultrasonics Sonochemistry, 21(4), 1578-1584. https://doi.org/10.1016/j.ultsonch.2014.01.0 24.

Mendonça, F. A. C. (2003, maio). Atividade larvicida de diferentes extratos de Annona glabra L. frente às larvas de Aedes aegypti L. (Diptera: Culicidae). Apresentado em XXVI Reunião Anual da Sociedade Brasileira de Química, Poços de Caldas, Minas Gerais. Recuperado de http://www.sbq.org.br/26ra/.

Miyoshi, H., Ohshima, M., Shimada, H., Akagi, T., Iwamura, H., \& McLaughlin, J. L. (1998). Essential structural factors of annonaceous acetogenins as potent inhibitors of mitochondrial complex I. Biochimica et Biophysica Acta (BBA) - Bioenergetics, 1365(3), 443-452. https://doi.org/10.1016/S00052728(98)00097-8.

Morré, D. J., de Cabo, R., Farley, C., Oberlies, N. H., \& McLaughlin, J. L. (1995). Mode of action of bullatacin, a potent antitumor acetogenin: Inhibition of NADH oxidase activity of HeLa and HL-60, but not liver, plasma membranes. Life Sciences, $56(5)$, 343-348. https://doi.org/10.1016/00243205(94)00957-0.

NAP. (1989). Read "Lost Crops of the Incas: Little-Known Plants of the Andes with Promise for Worldwide Cultivation" at NAP.edu. Washington, DC: National Academies Press (https://www.nap.edu/read/1398/chapter/1). https://doi.org/10.17226/1398.

Ohsawa, K., Atsuzawa, S., Mitsui, T., \& Yamamoto, I. (1991). Isolation and Insecticidal Activity of Three Acetogenins from Seeds of Pond Apple, Annona glabra L. Journal of Pesticide Science, 16(1), 93-96. https://doi.org/10.1584/jpestics.16.93.

Padmaja, V., Thankamany, V., Hara, N., Fujimoto, Y., \& Hisham, A. (1995). Biological activities of Annona glabra. Journal of Ethnopharmacology, 48(1), 21-24. https://doi.org/10.1016/03788741(95)01277-K.

Pino, J. A., Marbot, R., \& Agüero, J. (2002). Volatile Components of Baga (Annona glabra L.) Fruit. Journal of Essential Oil Research, 14(4), 252-253. https://doi.org/10.1080/10412905.2002.9699 844.

Pontes, A. F., Barbosa, M. R. de V., \& Maas, P. J. M. (2004). Flora Paraibana:
Annonaceae Juss. Acta Botanica Brasilica, $18(2)$, 281-293. https://doi.org/10.1590/S010233062004000200008.

Rocha, G. S., Matsumoto, R. S., Lombardi, A. T., Lima, M. I. S., Rocha, G. S., Matsumoto, R. S., \& Lima, M. I. S. (2017). Potential effects of fungicide and algaecide extracts of Annona glabra L. (Annonaceae) on the microalgae Raphidocelis subcapitata and on the oomycete Pythium. Anais Da Academia Brasileira de Ciências, 89(3), 2101-2111. https://doi.org/10.1590/00013765201720160040.

Santos, A. S., Andrade, E. H. D. A., Zoghbi, M. D. G. B., \& Maia, J. G. S. (1998). Volatile constituents of fruits of Annona glabra L. from Brazil. Flavour and Fragrance Journal, 13(3), 148-150. https://doi.org/10.1002/(SICI)10991026(199805/06)13:3<148::AIDFFJ711>3.0.CO;2-Y.

Scherer, H. W. (1995). Larcher, W. (Hrsg.): Plant ecophysiology. Ulmer Verlag, Stuttgart 1994; 360p., DM 78. Journal of Plant Nutrition and Soil Science, 158(2), 207-207. ISBN 3-8252-8074-8. https://doi.org/10.1002/jpln.19951580214.

Silberbauer-Gottsberger, I., Gottsberger, G., \& Webber, A. (2003). Morphological and Functional Flower Characteristics of New and Old World Annonaceae with Respect to Their Mode of Pollination. Taxon, 52, 701718. https://doi.org/10.2307/3647345.

Thang, T. D., Dai, D. N., Hoi, T. M., \& Ogunwande, I. A. (2013). Study on the volatile oil contents of Annona glabra L., Annona squamosa L., Annona muricata L. and Annona reticulata L., from Vietnam. Natural Product Research, 27(13), 1232-1236.

https://doi.org/10.1080/14786419.2012.7244 13.

The angiosperm phylogeny group. APG (2003). An update of the Angiosperm Phylogeny Group classification for the orders and families of flowering plants: APG II. Botanical Journal of the Linnean Society, 141(4), 399-436. https://doi.org/10.1046/j.10958339.2003.t01-1-00158.x.

Tsou, C. H., \& Fu, Y.-L. (2002). Tetrad pollen formation in Annona (Annonaceae): Proexine formation andbinding mechanism. American Journal of Botany, 89(5), 734-747. https://doi.org/10.3732/ajb.89.5.734.

WHO. (1983). Report of the Scientific Working Group on Plant Molluscicides. Geneva, Switzerland: World Health Organization. 
Recuperado de https://apps.who.int/iris/bitstream/handle/10 665/60086/TDR_SCH-

SWG_4_83.3_eng.pdf?sequence $=1 \&$ isAllo wed $=$ y.

WHO. (1996). Report of the WHO Informal Consultation on the "Evaluation and Testing of Insecticides", WHO/HQ, Geneva, 7 to 11 October 1996. Recuperado de https://apps.who.int/iris/handle/10665/659\}

Wu, T., He, M., Zang, X., Zhou, Y., Qiu, T., Pan, S., \& Xu, X. (2013). A structure-activity relationship study of flavonoids as inhibitors of $\mathrm{E}$. coli by membrane interaction effect. Biochimica et Biophysica Acta (BBA) Biomembranes, 1828(11), 2751-2756. https://doi.org/10.1016/j.bbamem.2013.07.0 29.

Zeng, L., Ye, Q., Oberlies, N. H., Shi, G., Gu, Z. M., He, K., \& McLaughlin, J. L.
(1996). Recent advances in Annonaceous acetogenins. Natural Product Reports, 13(4), 275-306. https://doi.org/10.1039/np9961300275.

Zhang, Y., Peng, H., Xia, G., Wang, M., \& Han, Y. (2004). Anticancer effect of two diterpenoid compounds isolated from Annona glabra Linn. Acta Pharmacologica Sinica, 25(7), 937-942.

Zotz, G, Harris., G., Königer, M., \& Winter, K. (1995). High rates of photosynthesis in the tropical pioneer tree, Ficus insipida Willd. Flora., 190(3), 265-272. https://doi.org/10.1016/s03672530(17)30663-1.

Zotz, G., Tyree, M., \& Patiño, S. (1997). Hydraulic architecture and water relations of a flood-tolerant tropical tree, Annona glabra. Tree Physiology, 17(6), 359-365. https://doi.org/10.1093/treephys/17.6.359. 